\title{
24. \\ Beiträge zur Theorie der kürzesten Linien auf krummen Flächen.
}

( Von Herrn Dr. Ferd. Minding zn Berlin.)

Aus der Formel für das Linear-Element, nämlich $d s^{2}=E d p^{2}+2 F d p d q$ $+G d q^{2}$, ergiebt sich für die kürzeste Linie auf einer krummen Fläche folgende Differential-Gleichung zweiter Ordnung zwischen $p$ und $q$, vämlich:

$$
\text { 1. } \frac{d E}{d p} d p^{2}+2 \frac{d F}{d p} d p d q+\frac{d G}{d p} d q^{2}=2 d s . d\left(\frac{E d p+F d q}{d s}\right) \text {. }
$$

Diese allgemeine Gleichung soll hier zunächst angewendet werden auf die abwickelbaren Flächen, im gewöhnlichen Sinne dieses Ausdruckes. Es seien $u, v, w$ die Coordinaten, $\varphi$ der Bogen der Knotenlinie einer solchen Fläche, sämmtlich Functionen einer Veränderlichen $p$, so lassen sich mit Hülfe der zweiten Veränderlichen $q$ die Coordinaten der Fläche ausdrücken wie folgt :

Man setze

$$
x=u+q \frac{d u}{d \varphi}, \quad y=v+q \frac{d v}{d \varphi}, \quad z=w+q \frac{d w}{d \varphi} .
$$

$$
\left(d \frac{d u}{d \varphi}\right)^{2}+\left(d \frac{d v}{d \varphi}\right)^{2}+\left(d \frac{d w}{d \varphi}\right)^{2}=d \boldsymbol{P}^{2}
$$

und bemerke, dafs $d u^{2}+d v^{2}+d w^{2}=d \Phi^{2}$ ist; so folgt für das LinearElement die Formel

$$
d s^{2}=q^{2} d P^{2}+(d q+d \varphi)^{2},
$$

oder

$$
\text { 2. } d s^{2}=(\boldsymbol{Q}-\boldsymbol{P})^{2} d \boldsymbol{P}^{2}+d \boldsymbol{Q}^{2} \text {, }
$$

wenn man eine neue Veränderliohe $Q$ einführt, nämlich :

$$
\boldsymbol{Q}=\boldsymbol{q}+\boldsymbol{\varphi}
$$

Schreibt man in (1.) $P$ anstatt $p, Q$ anstatt $q$, und setzt $E=(Q-\varphi)^{2}$, $F=0, G=1$, noch bemerkend, dafs $\varphi$ nur von $P$ abhängt, so erbält man folgende Differential-Gleichung der kürzesten Linie :

$$
\text { 3. }(Q-\varphi) \frac{d^{2} Q}{d P^{2}}-2\left(\frac{d Q}{d P}\right)^{2}+\frac{d \varphi}{d P} \cdot \frac{d Q}{d P}-(Q-\varphi)^{2}=0 \text {. }
$$

Von dieser erhält man, am leichtesten durch eine geometrische Betrachtung, folgendes Integral:

$$
\text { 4. }(\boldsymbol{Q}-\phi) \cos (\boldsymbol{P}-a)+\int d \varphi \cdot \cos (\boldsymbol{P}-a)=k \text {. }
$$


$a$ und $k$ sind willkürliche Constanten. Der Bogen $(\sigma)$ der kürzesten Linie wird ausgedrückt wie folgt:

$$
\sigma=q \sin (\boldsymbol{P}-a)+\int d \varphi \cdot \sin (\boldsymbol{P}-a)+\text { Const. }^{\prime}
$$

wo wieder $q$ für $\boldsymbol{Q}-\emptyset$ gesetzt ist.

Für die Curve vom kürzesten Umringe auf der abwickelbaren Fläiche gilt folgende Differential-Gleichung :

$$
\text { 5. }(Q-\emptyset) \frac{d^{2} Q}{d P^{2}}-2\left(\frac{d Q}{d P}\right)^{2}+\frac{d Q}{d P} \cdot \frac{d \varphi}{d P}-(Q-\varphi)^{2}=\frac{1}{h}\left(\frac{d s}{d P}\right)^{3} \text {. }
$$

Zur Abkürzung sei

$$
m+\int^{p} d \varphi \cdot \cos p=U, \quad n+\int^{p} d \varphi \cdot \sin p=V
$$

( $m$ und $n$ sind beliebige Constanten). Alsdann ist das Integral von (5.) in folgender Gleichung enthalten :

$$
\text { 6. } \quad(q+U \cos p+V \sin p)^{2}+(U \sin p-V \cos p)^{2}=h^{2} \text {. }
$$

Da $\boldsymbol{P}$ und $\varphi$ blols von der Veränderlichen $p$ abhängen und $\frac{d P}{d p}, \frac{d \varphi}{d p}$ als Functionen von $p$ gegeben sind, so ist durch vorstehende Formeln das Problem der kürzesten Linien, so wio der Curven kürzesten Umringes auf abwickelbaren Flächen, auf Quadraturen gebracht und mithịn allgemein gelöset.

Dafs auf jeder Fläche von unveränderlichem positivem Krümmunggmaafse zwischen den Seiten und Winkeln eines aus kürzesten Linien gebildeten Dreiecks die Formeln der sphärischen Trigonometrie gelten, folgt sogleich, wenn man sich erinnert, dafs jede Fläche dieser Art sich auf eine Kugel abwickeln läfst. Ist das Krümmungsmaafs negativ, so gelten dieselben Formeln mit der Aenderung, dafs die hyperbolischen Functionen der Seiten an die Stelle der trigonometrischen treten. Sind nämlich $a, b, c$ die Seiten des Dreieckes, $\boldsymbol{A}$ der Gegenwinkel von $a$, und $k$ das unveränderliche Krümmungsmaafs, gleichviel ob positiv oder negativ, so ist es nicht schwer, die Richtigkeit folgender Gleichung zu beweisen:

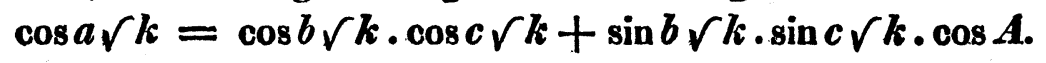

Im 19ten Bande dieses Journals, Seite 379, findet man eine Gruppe von Flächen von unveränderlichem negativem Krümmungsmaafse $(k=-1)$ angegeben, deren Linear-Element daselbst in folgender Form dargestellt wird :

$$
d s^{2}=\left(v^{2}+h^{2}-a\right) d t^{2}+\frac{d v^{2}}{v^{2}+h^{2}-a}
$$


Nimmt man auf einer solchen Fläche einen beliebigen Punct $\boldsymbol{A}$, dessen Argumente $v=v^{\prime}, t=t^{\prime}$ seien, und zieht aus $A$ nach einem zweiten zu $v$ und $t$ gehörigen Puncte $B$ eine kürzeste Linie auf der Fläche, setzt die Länge ihres Bogens $\boldsymbol{A B}=\sigma$, den Winkel, unter welchem sie in $A$ die Curve von unveränderlichen $v$ schneidet, $=\theta$; so gelten zwischen den Veränderlichen $v, t, \sigma, \theta$ folgende Gleichungen, in welchen $r\left(a-h^{2}\right)=b$ gesetzt ist, nämlich :

7. $\left\{\begin{array}{c}\frac{b v^{\prime} \operatorname{cotg} \theta-b^{2} \operatorname{tang} b\left(t-t^{\prime}\right)}{b+v^{\prime} \operatorname{cotg} \theta \cdot \operatorname{tang} b\left(t-t^{\prime}\right)}=\frac{\cos \theta V\left(v^{\prime 2}-b^{2}\right) \cdot\left(v^{\prime}+\mathfrak{L a n g} \sigma \cdot \sin \theta V\left(v^{\prime 2}-b^{2}\right)\right)}{\sin \theta V\left(v^{\prime 2}-b^{2}\right)+v^{\prime} \mathfrak{T a n g} \sigma} . \\ v=\operatorname{Sin} \sigma \cdot \sin \theta \cdot V\left(v^{\prime 2}-b^{2}\right)+v^{\prime}\left(\operatorname{Sog}_{0} \sigma .\right.\end{array}\right.$

Diese Formeln gelten ohne Ausnahme, die Grölse $b^{2}=a-h^{2}$ mag positiv, Null, oder negativ sein. Durch sie verwandelt sich der obige Werth von $d s^{2}$ in $d s^{2}=d \sigma^{2}+\operatorname{Sin}^{2} \cdot d \theta^{2}$, wie erforderlich. Man kann sie auch leicht auf eine einfachere Gestalt bringen; denn wenn z. B. $a-h^{2}$ negativ ist, so setze man :

$$
\begin{array}{rc}
v=r\left(h^{2}-a\right) \operatorname{Sin} q, & \checkmark\left(h^{2}-a\right) t=p, \\
v^{\prime}=\sqrt{ }\left(h^{2}-a\right) \operatorname{Sin} q^{\prime}, & \checkmark\left(h^{2}-a\right) t^{\prime}=p^{\prime}, \\
\mathfrak{T a n g} w=\operatorname{cotg} \theta . \operatorname{Sin} q^{\prime}, & \text { Tang } u=\frac{\mathfrak{I a n g} q^{\prime}}{\sin \theta},
\end{array}
$$

wo die Hülfsgröfren $w$ und $u$ einen imaginären Theil haben können, der nachber wieder aus der Rechnung herausfällt. Dadurch gehen die Gleichungen 7. in folgende über:

$$
\begin{aligned}
\operatorname{sang}\left(p-p^{\prime}+w\right) & =\cos \theta \cdot \operatorname{Sog} q^{\prime} \cdot \operatorname{sang}(\sigma+u), \\
\operatorname{Sin} q \cdot \operatorname{Sin} u & =\operatorname{Sin} q^{\prime} \cdot \operatorname{Sin}(\sigma+u), \\
\operatorname{Sog} q \cdot \operatorname{Sog}\left(p-p^{\prime}+w\right) & =\operatorname{Sog}(\sigma+u),
\end{aligned}
$$

von welchen die letzte aus den beiden ersten folgt. Ist $a-h^{2}=0$, so kommt

$$
v\left(t-t^{\prime}\right)=\operatorname{Sin} \sigma \cdot \cos \theta, \quad v=v^{\prime}(\operatorname{Cog} \sigma+\operatorname{Sin} \sigma \sin \theta) \text {. }
$$

Versteht man unter $p$ und $q$ die Argumente der Krümmungslinien auf einem Ellipsoïd, dessen Gleichung sei : $\frac{x^{2}}{a^{2}}+\frac{y^{2}}{b^{2}}+\frac{z^{2}}{c^{2}}=1$, so ist $\frac{x^{2}}{a^{2}}=\frac{\left(a^{2}-p\right)\left(a^{2}-q\right)}{\left(a^{2}-b^{2}\right)\left(a^{2}-c^{2}\right)}, \quad \frac{y^{2}}{b^{2}}=\frac{\left(b^{2}-p\right)\left(b^{2}-q\right)}{\left(b^{2}-a^{2}\right)\left(b^{2}-c^{2}\right)}, \quad \frac{z^{2}}{c^{2}}=\frac{\left(c^{2}-p\right)\left(c^{2}-q\right)}{\left(c^{2}-a^{2}\right)\left(c^{2}-b^{2}\right)}$. Hieraus folgt

wo gesetzt ist:

$$
d s^{2}=\frac{1}{1}(p-q)\left(\boldsymbol{P} d p^{2}-\boldsymbol{Q} d q^{2}\right),
$$

$$
\boldsymbol{P}=\frac{p}{\left(a^{2}-p\right)\left(b^{2}-p\right)\left(c^{2}-p\right)}, \quad \boldsymbol{Q}=\frac{q}{\left(a^{2}-q\right)\left(b^{2}-q\right)\left(c^{2}-q\right)} .
$$

Crelle's Journal d. M• Bd. XX. Hft. 4. 
Zur Abkürzung sei noch

$$
\frac{P}{Q}=\lambda \text {. }
$$

Für die kürzeste Linie erhält man nun folgende Differential-Gleichung :

8. $(\lambda d p-d q)\left(\lambda d p^{2}-d q^{2}\right)+(p-q) d q\left(d \lambda d p+2 \lambda d^{2} p\right)=0$, worin $d^{2} q=0$ gesetzt ist. Der Anblick dieser Gleichung läfst kaum eine so einfache Integration erwarten, wie sie Herr Prof. Jacobi im 19ten Bande Seite 309 mitgetheilt hat. Man kann dieses merkwürdige Integral auf folgende Weise aus (8.) herleiten :

Multiplicirt man die Gleichung (8.) mit $d p$ und bemerkt, dafs $d p\left(d \lambda d p+2 \lambda d^{2} p\right)=d\left(\lambda d p^{2}\right)=d\left(\lambda d p^{2}-d q^{2}\right)$ ist, so erhält man $(\lambda d p-d q)\left(\lambda d p^{2}-d q^{2}\right) d p+(p-q) d q d\left(\lambda d p^{2}-d q^{2}\right)=0$.

Diese Gleichung werde mit $d q$ multiplicirt und folgende identische bemerkt:

$$
(\lambda d p-d p) d p d q=d\left(\lambda q d p^{2}-p d q^{2}\right)-q d\left(\lambda d p^{2}-d q^{2}\right),
$$

so findet man

$$
\left(\lambda d p^{2}-d q^{2}\right) d\left(\lambda q d p^{2}-p d q^{2}\right)=\left(\lambda q d p^{2}-p d q^{2}\right) d\left(\lambda d p^{2}-d q^{2}\right),
$$

aus welcher sofort das Integral mit der willkürlichen Constante $h$ hervorgeht, nämlich :

oder

$$
\lambda q d p^{2}-p d q^{2}=h\left(\lambda d p^{2}-d q^{2}\right)
$$

$$
\text { 9. } \frac{P \cdot d p^{2}}{p-h}=\frac{Q \cdot d q^{2}}{q-h} \text {. }
$$

Durch Addition der Gleichungen

$$
\begin{aligned}
4 d s^{2} & =(p-q)\left(\boldsymbol{P} d p^{2}-\boldsymbol{Q} d q^{2}\right), \\
0 & =\{r(q-h \cdot \boldsymbol{P}) \cdot d p-\checkmark(p-h \cdot \boldsymbol{Q}) \cdot d q\}^{2}
\end{aligned}
$$

ergiebt sich nach Ausziehung der Wurzel für den Bogen der kürzesten Linie :

$$
\text { 10. } 2 d s=\sqrt{ }(p-h \cdot \boldsymbol{P}) \cdot d p-V(q-h \cdot Q) \cdot d q \text {. }
$$

Denkt man sich $a^{2}>b^{2}>c^{2}$, so liegt von den Argumenten $p, q$ das eine immer zwischen $a^{2}$ und $b^{2}$, das andere zwischen $b^{2}$ und $c^{2}$; die Constante $h$ in (9.) liegt nothwendig zwischen $p$ und $q$. Es sei $p>q$, so bewegt sich, wenn $h>b^{2}$ ist, in den vorstehenden Gleichungen $p$ immer zwischen $a^{2}$ und $h$; hingegen $q$ durchläuft alle Werthe von $b^{2}$ bis $c^{2}$. Für $p=h$ wird in (9.) $d p=0$, d. h. die Curve berührt die Krümmungslinie, oder vielmehr die beiden Krümmungslinien, für welche $p=h$ ist. Für $p=a^{2}$ wird gleichfalls $d p=0$, also $p$ für einen Augenblick unveränderlich; d. h. die Curve geht, indem sie den Hauptsohnitt, für welchen $p=a^{2}$ ist, schneidet, 
von einer Krümmungslinie, in unendlicher Nähe dieses Hauptschnittes, zu einer anderen demselben Werthe von $p$ entsprechenden über. Aehnlich verhält es sich, wenn $h$ zwischen $b^{2}$ und $c^{2}$ liegt; alsdann berührt die Curve abwechselnd die beiden zu $q=h$ gehörigen Hauptschnitte. Sohreibt man in den vorstehenden Formeln $-c^{2}$ für $c^{2}$, so ergeben sich die Ausdrücke für kürzeste Linien auf dem einfachen Hyperboloïd, in welchen $p$ zwischen $a^{2}$ und $b^{2}, q$ zwischen $-c^{2}$ und $-\infty$ liegen mufs. Für $h=0$ giebt die Gleichung (9.) die geraden Linien auf dieser Fläche. Schreibt man noch $-b^{2}$ für $b^{2}$, so erhält man die Ausdrücke für das zweitheilige Hyperboloï.

Um die Ausdrücke für das Paraboloïd zu erhalten, schreibe man in den Formeln für das Ellipsoïd $z-c$ statt $z, m c$ statt $a^{2}, n c$ statt $b^{2}, p c$, $q c$, $k c$ statt $p, q, h$, und setze hierauf $c=\infty$, so kommt

$$
\begin{aligned}
\frac{x^{2}}{m} & =\frac{(m-p)(m-q)}{n-m}, \quad \frac{y^{2}}{n}=\frac{(n-p)(n-q)}{m-n}, \quad 2 z=p+q-m-n, \\
4 d s^{2} & =(p-q)\left\{\frac{p d p^{2}}{(m-p)(n-p)}-\frac{q d q^{2}}{(m-q)(n-q)}\right\} .
\end{aligned}
$$

Die Gleichungen für die kürzeste Linie und die für den Bogen derselben sind

$$
\begin{gathered}
\frac{p \cdot d p^{2}}{(p-m)(p-n)(p-h)}=\frac{q \cdot d q^{2}}{(q-m)(q-n)(q-h)}, \\
2 d s=\sqrt{\left(\frac{p(p-h)}{(p-m)(p-n)}\right) d p-V\left(\frac{q(q-h)}{(q-m)(q-n)}\right) d q .}
\end{gathered}
$$

Die Ausdrücke für den Kegel und den Cylinder zweiten Grades sind in den oben allgemein für abwickelbare Flächen gegebenen enthalten. Ich bemerke noch, dafs die dort eingeführten Argumente $\boldsymbol{P}$ und $\boldsymbol{Q}$ ebenfalls wieder den Krümmungslinien entsprechen; nämlich $\boldsymbol{P}$ den geraden Linien der Fläche und $\boldsymbol{Q}$ dem anderen Systeme von Krümmungslinien. 